\title{
Effects of ultra-long fermentation time on the microbial community and flavor components of light-flavor Xiaoqu Baijiu based on fermentation tanks
}

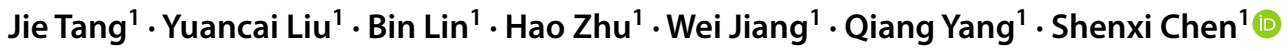

Received: 3 August 2021 / Accepted: 29 October 2021 / Published online: 24 November 2021

(c) The Author(s), under exclusive licence to Springer Nature B.V. 2021

\begin{abstract}
Microbial structure and succession of fermented grains play a significant role in Baijiu's flavor and quality. In this study, high-throughput sequencing (HTS) coupled with headspace solid-phase microextraction-gas chromatography-mass spectrometry (HS-SPME-GC-MS) were used to analyze the microbial community structures and flavor components in the fermented grains at the end of fermentation from different fermentation time of light-flavor Xiaoqu Baijiu. HTS results showed that Lactobacillus acetotolerans, Lactobacillus helveticus, Lactobacillus buchneri, Wickerhamomyces, Saccharomyces, and Condenascus were identified as the dominant microbes, but Lactobacillus (96.28\%) exhibited obvious advantages at the end of ultra-long fermentation time (day 98). HS-SPME-GC-MS analysis revealed that esters and alcohols had the most abundance in fermented grains of day 98, containing high concentrations of ethyl acetate, diethyl succinate, phenylethyl alcohol, isoamyl alcohol, and n-propanol, which were related to the succession of Lactobacillus and yeast communities. Interestingly, the content of n-propanol in the ultra-long fermentation time samples (day 98) was 6 times of that in normal fermented grains (day 14), which may be caused by higher abundance of Lactobacillus in day 98 samples. Monte Carlo permutation test showed residual starch, acidity, and amino nitrogen $(p<0.05)$ were important factors affecting the microbial community. Together, these results shed light on the physicochemical changes, microbial dynamics, and key flavor components of fermented grains at the end of fermentation from different fermentation time and provide a strategy for further improvement of Baijiu quality.
\end{abstract}

Keywords Baijiu $\cdot$ Fermented grains $\cdot$ Microbial community $\cdot$ Physicochemical indexes $\cdot$ Volatile compounds

\section{Introduction}

Baijiu, as the national liquor of China, with a 2000-year history, is a type of traditional distilled spirit drink produced by spontaneous mixed solid-state fermentation. Baijiu is the world's most-consumed spirit, and the gross sales of Baijiu in 2019 were more than $\$ 80$ billion (Ye et al. 2021). The traditional Baijiu process mainly includes material preparation, Jiuqu (starter) preparation, alcoholic fermentation, solid state distillation, aging and blending (Jin et al. 2017). As

Qiang Yang

yq@jingpai.com

$\triangle$ Shenxi Chen

chenshenxi2006@163.com

1 Hubei Provincial Key Lab for Quality and Safety of Traditional Chinese Medicine Health Food, Jing Brand Research Institute, Jing Brand Co., Ltd., Daye 435100, Hubei, People's Republic of China
Jiuqu preparation and alcoholic fermentation are carried out under partially controlled conditions, the specific microflora has been well enriched with a unique ecological environment and production procedures through repeated practices over a long time. The specific microbiota along with different materials and processing procedures have created different types of Baijiu. Based on the flavor characteristics, Baijiu fell into three major types, sauce-flavor, strong-flavor, and light-flavor (Zheng and Han 2016). The light-flavor Ваijiu brewing process takes place in ceramic jars, not the mud used in the other two types of Baijiu. The importance of fermentation pits and clay jars have been recognized by practice for thousands of years (Ye et al. 2021). Jiuqu, the fermentation starter of Baijiu, plays an important role in the fermentation process. According to the production process, Jiuqu includes Daqu (Made with raw wheat, barley and/or peas), Xiaoqu (Made with rice or rice bran), and Fuqu (Made with bran) (Jin et al. 2017). Light-flavor Xiaoqu Baijiu is produced by solid-state fermentation of sorghum with Xiaoqu (starter) 
containing various microbes and their complex interactions, which is a representative product made by Jing Brand Co., Ltd., one of the largest and most influential manufacturers of Xiaoqu Baijiu based on mechanized technology in China (Hu et al. 2021b). The quality evaluation standard of lightflavor Xiaoqu Baijiu mainly depends on sensory indexes and varieties and contents of core flavor compounds, which are associated with microbial metabolism during brewing process. Therefore, profiling the composition and metabolic activities of microbial community in fermented grains during the mechanized production is necessary to improve the quality of light-flavor Xiaoqu Baijiu.

Like all naturally fermented foods, the change in microbial composition and succession during the brewing process is critical to the development and final quality of Baijiu (Huang et al. 2020; Zhao et al. 2019; Tan et al. 2019). During the natural brewing process of various types of Baijiu, the microbial community diversity and structure are affected by some environmental factors, such as acidity, moisture, temperature, substrates, and fermentation time (Zhang et al. 2021; Hao et al. 2021). For instance, Zhang et al. (2021) found that altering the initial temperature of fermentation process led to differences in microbial succession rates and volatile flavors in the fermented grains of sauce-flavor Baijiu. Guan et al. (2020) revealed that changes in starch, moisture, and $\mathrm{pH}$ had significant effects on bacterial composition in strong-flavor Baijiu brewing process. Fan et al. (2020) expounded that the physicochemical properties changed continuously during aging of Daqu to provide a high esterifying activity and helped to stabilize the flavor compounds in mature Daqu. Thus microorganisms and environmental factors are important factors affecting the formation of flavor components in Baijiu. Hu et al. (2021c) and Dong et al. (2020) studied the microbial communities and identified the core microorganisms related to higher alcohol formation in two kinds of light-flavor Xiaoqu Baijiu brewing process (traditional and new mechanical technologies). However, there are few research to reveal the relationship between microbial composition, physicochemical indexes, and volatile components of light-flavor Хiaoqu Вaijiu brewing process.

Light-flavor Xiaoqu Baijiu produced based on mechanized technology is fermented in a truck-shaped tank $(1.7 \mathrm{~m} \times 1.0 \mathrm{~m} \times 1.0 \mathrm{~m})$ for 14 days with sorghum as the raw material and Xiaoqu as the starter (Jiang et al. 2019). Figure 1 shows the process flow chart of the detailed mechanized technology for the brewing process. The fermentation time was usually extended to 27 days due to vacations during the Chinese New Year. But due to COVID-19, people's work and life were severely affected and had to stay at home, which led to the delay in resuming work and production. So the fermentation period had been extended by 68-84 days, which was rare in a century. And so far, there are almost no research on the effects of ultra-long fermentation time on microbial composition, physicochemical factors, and flavor components in fermented grains. Lee et al. (2019) revealed the effect of long-term fermentation on volatile compounds in soy sauce and indicated that the contents of most compounds, such as acids, aldehydes, esters, lactones, and pyrazines, showed a tendency to increase, whereas those of alcohols and ketones decreased according to long-term fermentation. But it is unclear to what extent ultra-long

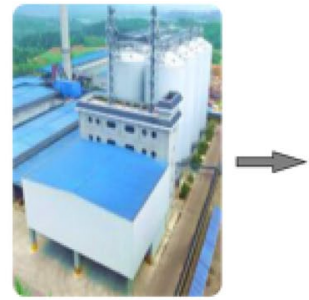

Granary

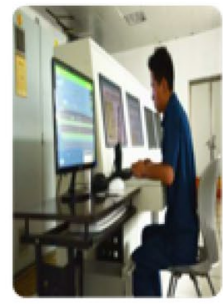

Central control system

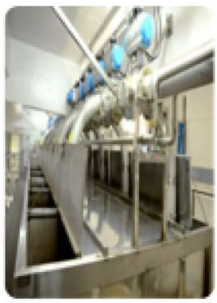

Grain-steeping
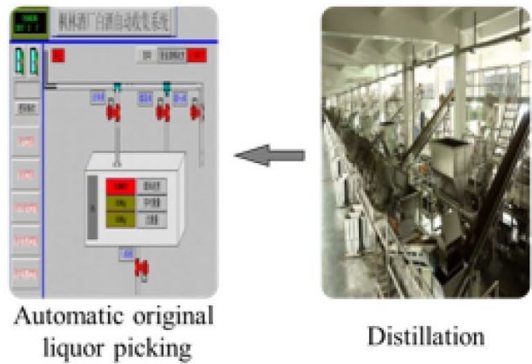

Distillation
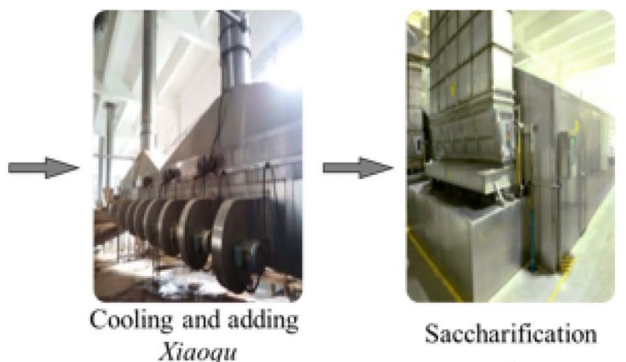

Saccharification

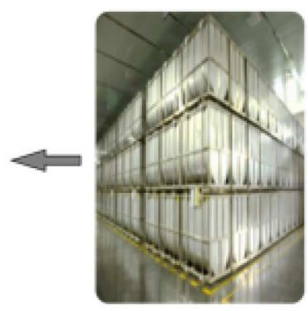

Fermentation

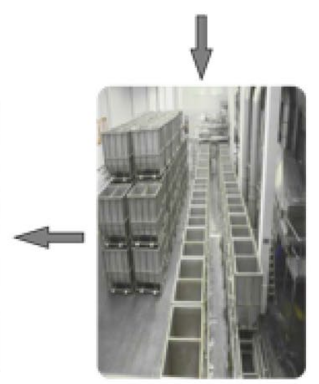

Blending with distilled grains

Fig. 1 Schematic of the mechanized brewing process of light-flavor Xiaoqu Baijiu 
fermentation time affects the microbial community in the grains and quality of Baijiu fermentation.

Therefore, the aim of this work was to determine the effects of ultra-long fermentation time on microbial community, physicochemical factors, and volatile components profiles during the light-flavor Xiaoqu Baijiu fermentation. And three typical fermented grains selected from the production process of light-flavor Xiaoqu Вaijiu based on mechanized technology with different fermentation time was subjected to investigation by high-throughput sequencing (HTS) and HP-SPME-GC-MS. To our knowledge, this is the first comprehensive study to report the effects of ultra-long fermentation time on its physicochemical properties, flavor components, and microbial community during the light-flavor Xiaoqu Baijiu fermentation. Together, our results reveal how ultra-long fermentation time affects the quality of Baijiu and provide a resource for further breeding of superior strains.

\section{Materials and methods}

\section{Sample collection and pretreatment}

Fermented grain samples were collected from a famous mechanized Baijiu factory (Hubei, China) in 2020. Samples $(250 \mathrm{~g})$ were collected from fermentation tanks in the middle layer (the depth of $0.5 \mathrm{~m}$ ) at the end of the fermentation period of 14, 87, and 98 days, and labeled as D14, D87, and D98, respectively. Each sample had three biological replicates. These samples were stored at $-20{ }^{\circ} \mathrm{C}$ for DNA extraction and volatile components analysis, and $4{ }^{\circ} \mathrm{C}$ for physicochemical properties analysis. Three independent batches were sampled for adequate representation.

\section{Microbiological and physicochemical analysis}

Changes in the physicochemical properties (moisture, residual starch, reducing sugar, acidity, and amino nitrogen) were measured as previous study described by Guan et al. (2020) and Fan et al. (2020). Moisture was determined by gravimetric method by drying fermented grain samples to a constant weight at $105^{\circ} \mathrm{C} .10 \mathrm{~g}$ of fermented grain samples were suspended with $90 \mathrm{~mL}$ ultrapure water and ultrasonically treated at $0{ }^{\circ} \mathrm{C}$ for $30 \mathrm{~min}$. The suspension was centrifuged at $4{ }^{\circ} \mathrm{C}$ for $10 \mathrm{~min}$ and the supernatant was pipetted to analyze the contents of acidity, amino nitrogen and reducing sugar. Acidity was determined by titration with $0.02 \mathrm{M} \mathrm{NaOH}$ yielding a titration endpoint of $\mathrm{pH}$ 8.2. Amino nitrogen was determined by titration with $0.05 \mathrm{~mol} / \mathrm{L} \mathrm{NaOH}$ yielding a titration endpoint of $\mathrm{pH} 9.2$ by adding $10 \mathrm{~mL}$ formaldehyde solution. Reducing sugar was analyzed using DNS method. Residual starch was calculated by calculating the difference between the total reducing sugar and the original reducing sugar after hydrolysis with $\mathrm{HCl}(20 \%$, v/v) for $30 \mathrm{~min}$.

Fermented grain samples $(10 \mathrm{~g})$ were put into an appropriate physiological salt solution to determine the population of microorganisms using selective media. Lactic acid bacteria (LAB) counts were performed on De Man, Rogosa, Sharpe Agar (MRSA) with $500 \mu \mathrm{g} / \mathrm{mL}$ of natamycin at $37^{\circ} \mathrm{C}$ for $48 \mathrm{~h}$. Yeasts and molds were enumerated on Rose Bengal Chloramphenicol Agar with $100 \mathrm{mg} / \mathrm{L}$ of chloramphenicol at $30{ }^{\circ} \mathrm{C}$ for $48-72 \mathrm{~h}$ (Huang et al. 2020). All physicochemical analyses were conducted in triplicate.

\section{DNA extraction, amplification, and sequencing}

DNA extraction, PCR amplification, and amplicon sequencing were conducted at Biomarker Bioinformatics Technology Co., Ltd (Nanjing, China). The total genomic DNA was extracted from $5 \mathrm{~g}$ of each sample according to previously published protocols (Amend et al. 2020) and purified using the Powersoil DNA Isolation Kit (MoBio, Carlsbad, CA, USA) following the manual. DNA concentration was quantified on a NanoDrop spectrophotometer (Thermo Scientific). The V3-V4 regions of the bacterial $16 \mathrm{~S}$ rDNA genes were amplified with the universal primers 338F (5'-ACTCCTACGGGAGGCAGCA- $3^{\prime}$ ) and 806R (5'-GGACTACHVGGGTWTCTAAT-3'). The internal transcribed spacer 1 (ITS1) region of the fungi was amplified with the forward primer ITS1F (5'-CTTGGTCATTTA GAGGAAGTAA-3') and the reverse primer ITS1R (5'GCTGCGTTCTTCATCGATGC-3') (Zang et al. 2018).

PCR was carried out in $50 \mu \mathrm{L}$ reaction system and thermal cycling conditions was as follows: initial denaturation at $95{ }^{\circ} \mathrm{C}$ for $5 \mathrm{~min}$, followed by 28 cycles of denaturation at $95{ }^{\circ} \mathrm{C}$ for $45 \mathrm{~s}$, annealing at $55^{\circ} \mathrm{C}$ for $50 \mathrm{~s}$, and extension at $72{ }^{\circ} \mathrm{C}$ for $45 \mathrm{~s}$, and a final extension at $72{ }^{\circ} \mathrm{C}$ for $10 \mathrm{~min}$. Three separate reactions were conducted to account for potentially heterogeneous amplification from the environmental template for each sample. PCR products were purified using AXYGEN Gel Extraction Kit (QIAGEN), and the concentrations were carefully assessed by Thermo Scientific NanoDrop 8000 UV-Vis Spectrophotometer (NanoDrop Technologies, Wilmington, DE). The 16S rDNA gene amplicons using PacBio sequencing platform (third-generation amplicon technology) and ITS 1 gene amplicons based on Illumina HiSeq deep sequencing platform (second-generation amplicon technology) were sequenced at Biomarker Bioinformatics Technology Co., Ltd., Nanjing, China. All sequencing data have been submitted to the Sequence Read Archive (SRA) of the National Center for Biotechnology Information (NCBI) with accession number PRJNA732254. 


\section{Volatile components analysis}

The volatile components in fermented grains were determined by HS-SPME-GC-MS conducted on an Agilent 7890B GC system coupled with an Agilent 5977B MSD (CA, USA). The $10 \mathrm{~g}$ of the fermented grain sample was added to $25 \mathrm{~mL}$ of sterilized ultrapure water and soaked overnight at $4{ }^{\circ} \mathrm{C}$. After ultrasonic treatment for $30 \mathrm{~min}$, the sample was centrifuged at $6500 \times g$ at $4{ }^{\circ} \mathrm{C}$ for $10 \mathrm{~min}$. The supernatant $(8 \mathrm{~mL})$ was transferred to a $20 \mathrm{~mL}$ vial containing $10 \mu \mathrm{L}$ of 2-ethyl hexanol (internal standard) and $5 \mathrm{~g}$ of sodium chloride. Volatile compounds were collected for $45 \mathrm{~min}$ at $50{ }^{\circ} \mathrm{C}$, followed by GC-MS analysis (Wang et al. 2020b). Volatile compounds were identified by matching with the NIST14.L spectrum database and their quantitative analysis was calculated by comparison with the area of the internal standard.

\section{Bioinformatics and statistical analysis}

The raw sequences were quality-filtered to obtain specific sequences with the Quantitative Insights Into Microbial Ecology (QIIME) software package. Sequences with $\geq 97 \%$ similarities were clustered into operational taxonomic units (OTUs) according to UCLUST (Bokulich et al. 2013) and the bacterial taxonomic information was assigned to all OTUs by searching against the Silva databases using the Ribosomal Database Project (RDP) classifier within QIIME (Quast et al. 2012). The fungal sequences were queried against the GenBank non-redundant nucleotide database (nt) in NCBI using local BLASTn. The MEGAN program was used to assign BLAST hits to taxa of the NCBI taxonomy. After removing non-fungal sequence reads, the fungal sequences were clustered into operational taxonomic units (OTUs) at a 97\% similarity level using Uclust (Huson et al. 2007). Alpha diversity indices (Chao1 richness and ACE richness estimators, Simpson and Shannon diversity indexes) reflecting the richness and the diversity of the microbial community were evaluated using the OTUs table in QIIME (Ramette 2007). Relative abundance of bacteria and fungi and proportions of volatile components in the samples were plotted using Origin 2018. To associate the correlations between the dominant microbiota and important flavor components, Spearman's correlation coefficient was calculated between the microbial genera and flavor components via IBM SPSS Statistics (version 19.0), and visualized as a correlation network with $|\rho|>0.8$ and $p<0.05$ in Cytoscape (v3.6.1) (Shannon et al. 2003; Liu et al. 2019). Redundancy analysis (RDA) and Monte Carlo permutation test were performed using the vegan packages in $\mathrm{R}$ to confirm the correlations between microbial community structures and physicochemical properties.

\section{Results}

\section{General investigation of microbiota and physicochemical properties}

The physicochemical properties and cultivable microbiota abundance of fermented grains characterized the process of fermentation and reflected the quality of the fermented grains, and provided the first insights into the microbial composition and metabolic changes, which offered a basis for further amplicon sequencing analysis (Huang et al. 2020). As shown in Fig. 2a, the moisture, acidity, and amino nitrogen contents increased, while the residual starch and reducing sugar contents decreased with the extension of fermentation time. The moisture of the fermented grains at the end of the normal fermentation time (day 14) remained around $71.81 \%$, and increased steadily to $73.00 \%$ in the sample of D98. The titratable acidity and amino nitrogen content increased from 0.91 to $1.49 \mathrm{mmol} / 10 \mathrm{~g}$ and from 0.38 to $0.51 \mathrm{~g} / \mathrm{kg}$ from the samples D14 to D98, respectively. Both of them showed a quick increasing tendency during the extension of fermentation time from D14 sample to D87 sample, followed by a substantially increasing from D87 sample to D98 sample. Conversely, a significant decrease of the reducing sugar and residual starch contents could be observed from D14 sample to D98 sample. Moreover, the reducing sugar decreased rapidly from 2 to $0.83 \mathrm{~g} / 100 \mathrm{~g}$, and the residual starch contents maintained a stable range from D87 sample to D98 sample. The more thorough fermented grains caused by the extended fermentation time resulted in the changes of these physicochemical indicators.

The total numbers of fungi, yeasts, and LAB were 5.92, 4.65 and $7.36 \mathrm{lg}$ CFU/g in D14 sample, respectively (Fig. 2b). No molds were detected and the total numbers of yeasts declined with the extension of fermentation time in D87 and D98 samples. The total population of LAB was higher than that of fungi, and LAB had a relatively stable growth in D87 and D98 samples. The oxygen in the fermented grains was almost exhausted, and the acidity and alcohol content increased followed the extension of fermentation time, which led to the death of aerobic microbiota (molds, most types of yeasts) and survival of anaerobes and facultative anaerobic microorganisms (LAB).

\section{Microbial richness and diversity}

After filtering the low-quality reads and chimeras, there were a total of 33,087 effective sequences of 16S rDNA gene and 325,516 effective sequences of ITS1 rDNA gene to be obtained from all fermented grain samples (Table 1). Chao 1 and ACE indexes were used to estimate the microbial community richness, and Shannon index and Simpson index 
(a)
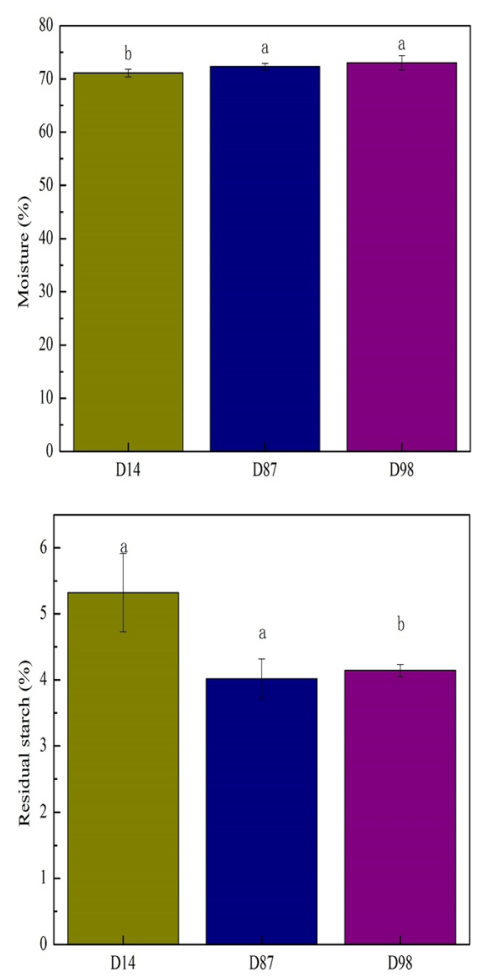

(b)

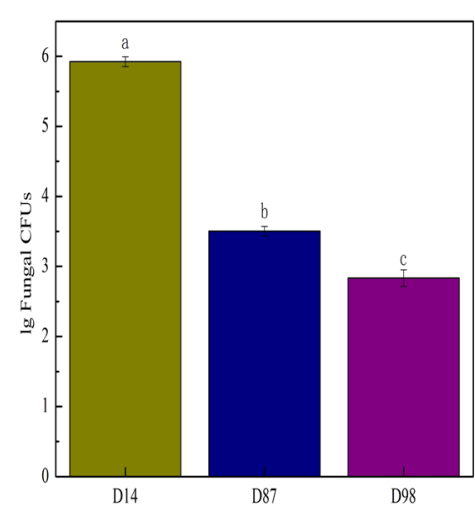

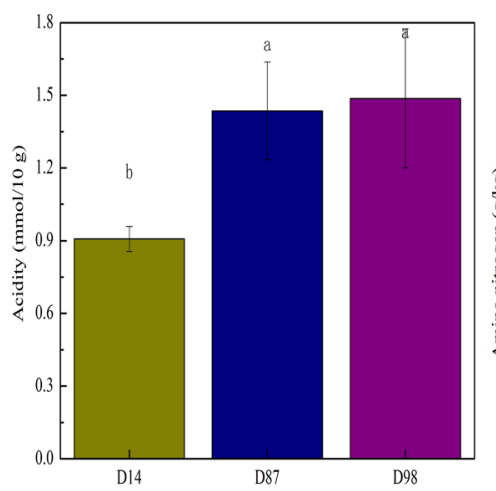
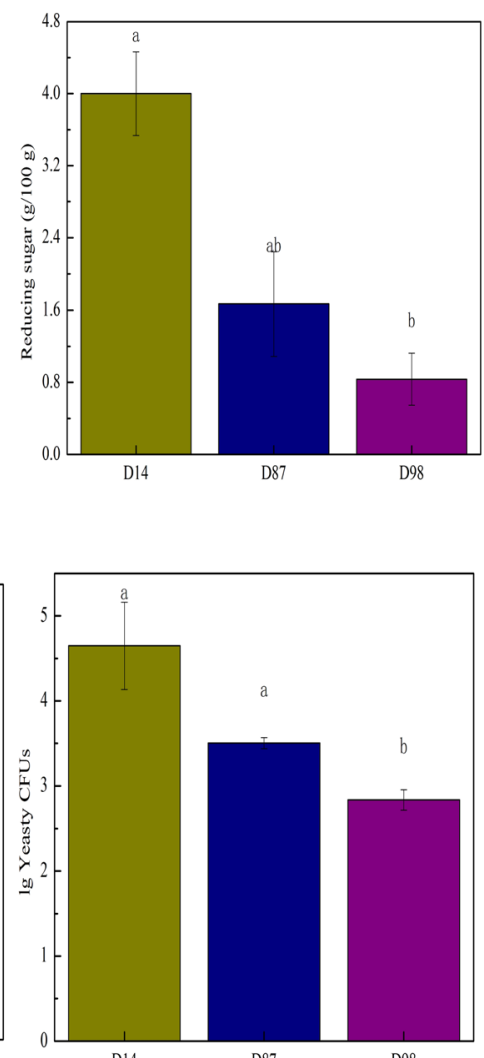

D98
$\frac{1}{\mathrm{D} 14}$

D87

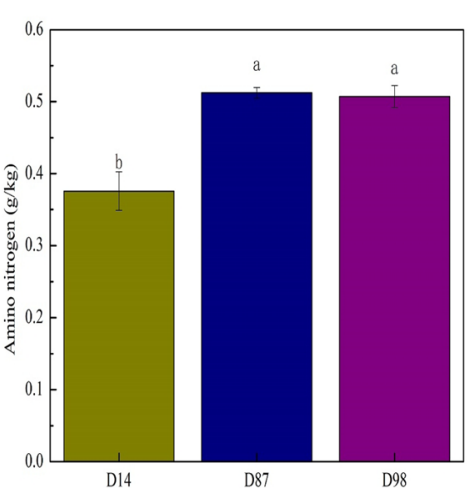

D87

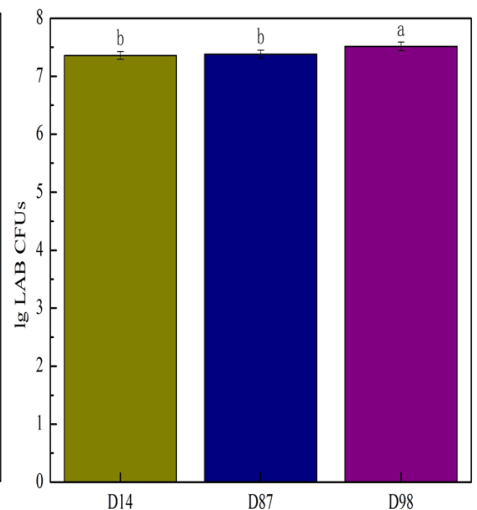

D98
Fig. 2 Dynamics of physicochemical characteristics and the viable microbial counts of the fermented grains at the end of different fermentation time (a Changes in the physicochemical characteristics of fermented grains; $\mathbf{b}$ Changes in the viable microbial counts of fermented grains. Different letters obtained by one-way ANOVA followed by the Duncan's test indicated significant differences at $p<0.05$ )
Table 1 Reads, OTUs and alpha-diversity indexes

\begin{tabular}{|c|c|c|c|c|c|c|c|}
\hline \multicolumn{2}{|c|}{ Sample ID } & \multirow[t]{2}{*}{ Reads } & \multirow[t]{2}{*}{ OTUs } & \multicolumn{4}{|c|}{ Alpha-diversity indexes } \\
\hline & & & & Simpson & Shannon & Chaol & ACE \\
\hline \multirow[t]{3}{*}{$16 \mathrm{~S}$} & D14 & 10,876 & 61 & $0.34 \pm 0.24$ & $1.20 \pm 0.70$ & $42.63 \pm 5.38$ & $43.83 \pm 4.34$ \\
\hline & D87 & 11,303 & 49 & $0.54 \pm 0.09$ & $1.99 \pm 0.29$ & $43.22 \pm 1.89$ & $52.62 \pm 3.28$ \\
\hline & D98 & 10,908 & 37 & $0.72 \pm 0.01$ & $2.49 \pm 0.10$ & $32.55 \pm 6.05$ & $41.24 \pm 11.57$ \\
\hline \multirow[t]{3}{*}{ ITS } & D14 & 98,454 & 346 & $0.82 \pm 0.02$ & $3.89 \pm 0.20$ & $329.09 \pm 11.97$ & $397.98 \pm 4.52$ \\
\hline & D87 & 124,100 & 342 & $0.92 \pm 0.03$ & $5.12 \pm 0.44$ & $328.12 \pm 17.38$ & $372.95 \pm 9.28$ \\
\hline & D98 & 102,962 & 368 & $0.94 \pm 0.04$ & $5.41 \pm 0.50$ & $366.93 \pm 68.68$ & $372.87 \pm 29.22$ \\
\hline
\end{tabular}


were used to evaluate the microbial community diversity. For bacterial communities, the richness estimators (Chao 1 and ACE) firstly increased from D14 to D87 and then decreased from D87 to D98 samples. But the microbial diversity (Simpson and Shannon) were observed to increase substantially with the extending fermentation time from D14, D87 to D98 samples. For fungal communities, the shift patterns of microbial richness and diversity were consistent with the OTUs indexes, both of which increased from D14, D87 to D98 samples.

\section{Microbial composition and dynamics}

The relative abundance of different bacterial microorganisms at the phylum level in all samples were shown in Fig. 3a. According to the sequences obtained from all samples, ten bacterial phyla were identified, and Firmicutes and Proteobacteria were the major phyla. There was a dramatic increase (from 62.5 to $96.4 \%$ ) in the proportion of Firmicutes during the extension of fermentation time from D14 to D87 samples, followed by a little decline from D87 to D98 samples, while the proportion of Proteobacteria was converse. Figure $3 b$ showed the relative abundance of fungi in all samples at the phylum level. All of the analyzed sequences were classified into 7 fungal phyla, mainly containing Ascomycota, Basidiomycota, Glomeromycota, and Mucoromycota. Among them, Ascomycota dominated and represented the major fungal phylum in all samples, which slightly decreased (88.9-82\%) from D14 to D98 samples. Ascomycota was prevalent in the production of fermented alcoholic beverages (Li et al. 2011). In general, the bacterial and fungal diversities at the phylum level were relatively simple (Zang et al. 2018).

Bacterial compositions at the species level and fungi at the genus level were determined to get a more detailed analysis of the bacterial and fungal dynamics associated (a)

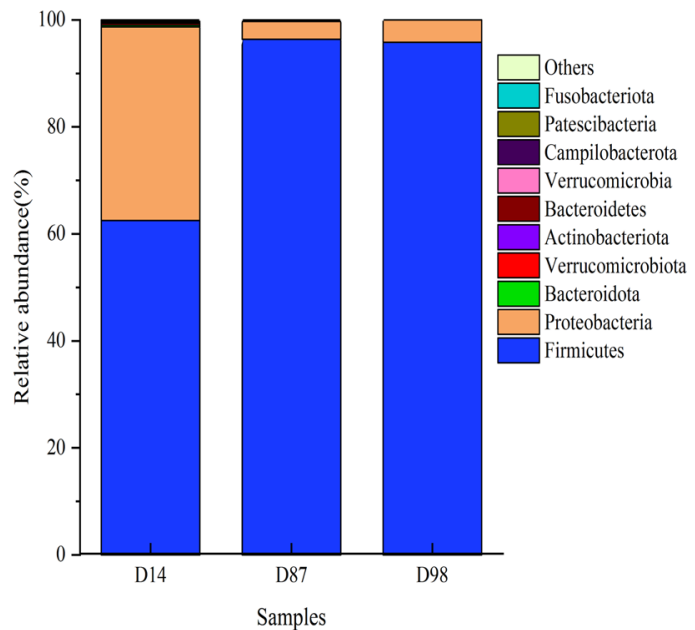

(c)

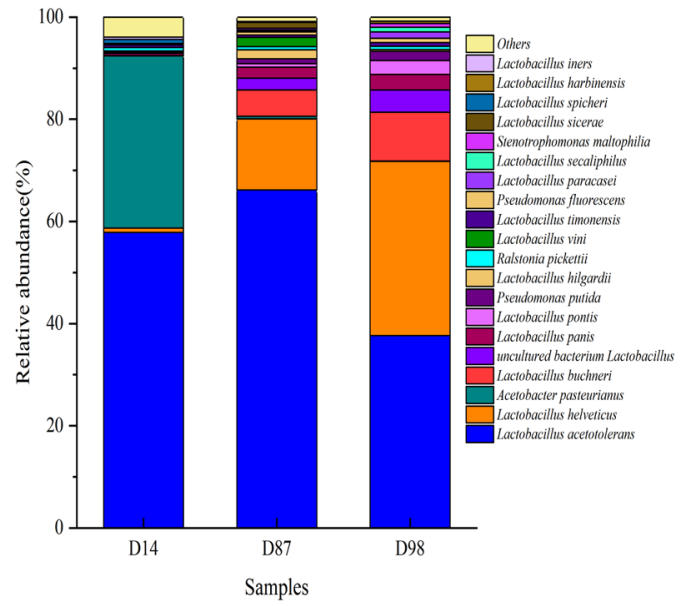

(b)

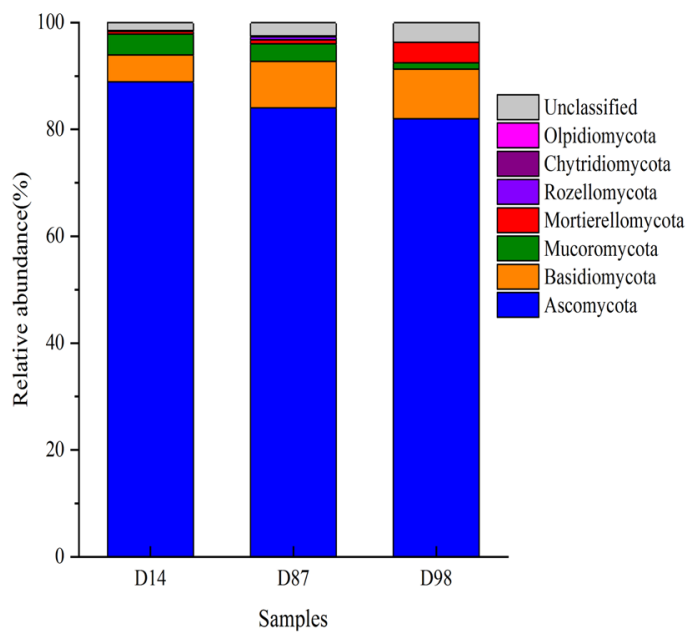

(d)

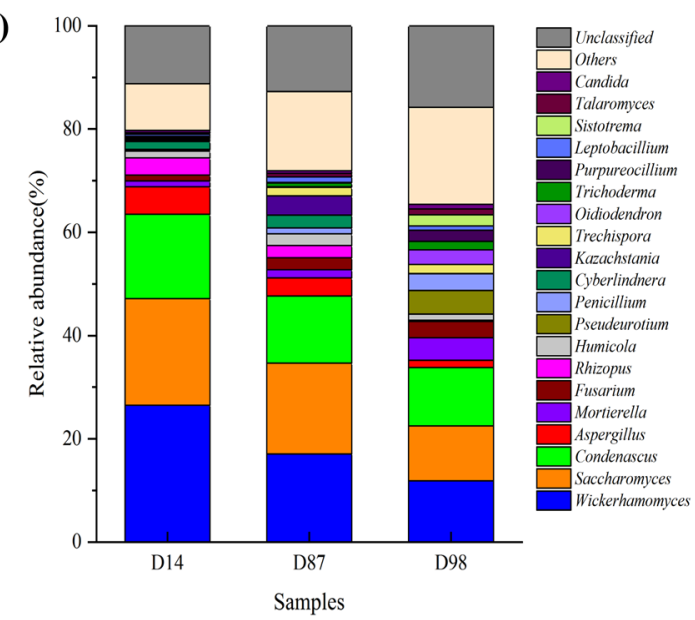

Fig. 3 Relative abundance of bacteria (a) and fungi (b) at the phylum level and bacteria (c) at the species and fungi (d) at the genus level in the samples 
with fermented grain samples. At the species level, the top 20 species were shown in Fig. 3c. Lactobacillus acetotolerans and Acetobacter pasteurianus were the predominant microorganisms in the fermented grains at the end of the normal fermentation time (D14). With the continuation of fermentation, A. pasteurianus disappeared and the abundance of $L$. acetotolerans (66.2\%) was the largest in D87 sample. In addition, the proportion of Lactobacillus helveticus and Lactobacillus buchneri gradually increased in D87 sample. Furthermore, Lactobacillus dominated at the end of the fermentation period (96.28\%), which suggested that members of Lactobacillus probably played key roles in the late fermentation of Baijiu. Some researchers have reported that Lactobacillus could produce several antimicrobial metabolites, including organic acids, which was related to the metabolism of amino acids during food fermentation processes and improved texture and flavor of fermented food (Quijada et al. 2017; Sudhanshu et al. 2018).

For fungal community structure, the top 20 genera were showed in Fig. 3d. The fungal community was dominated by these genera Wickerhamomyces (26.5\%), Saccharomyces (20.6\%), and Condenascus (16.3\%) in D14 sample, then decreased gradually with the fermentation period going on. Similarly, the relative abundance of Aspergillus, Rhizopus decreased from 5.3 to $1.4 \%$ and from 3.3 to $0.2 \%$ (from D14 to D98), respectively. Whereas the opposite dynamics of Fusarium, Pseudeurotium, Penicillium, Trichoderma, Purpureocillium, Talaromyces, and others were observed, which increased along with the fermentation time. Some studies have reported that yeast commonly contributes to the flavor and aroma of fermented products, such as Wickerhamomyces is conducive to the synthesis of ester compounds in the Chinese Baijiu brewing process (Wang et al. 2020a), while Saccharomyces is the most efficient ethanol producer during the Baijiu fermentation stage. Based on sensory evaluation, Baijiu produced by Saccharomyces is characterized by high scores for harmony and the highest values of aroma compared with other yeasts (Liu et al. 2017; Cai et al. 2018). Condenascus, a newly discovered genus in the fermented grains of Baijiu was identified, formerly belonging to the classification of Thielavia (Wang et al. 2019a).

\section{Analysis of volatile components in fermented grains}

About 64 major volatile compounds in fermented grains were identified by HS-SPME-GC-MS, and 31, 48, and 55 major components were found in D14, D87, and D98 samples, respectively. With the extension of the fermentation time, the types of volatile substances increased, especially from D14 sample to D87 sample. All volatile components were composed of six categories, including esters (25), alcohols (17, except ethanol), acids (7), phenols (6), aldehydes and ketones (4), and others (5). Figure 4 showed that esters accounted for

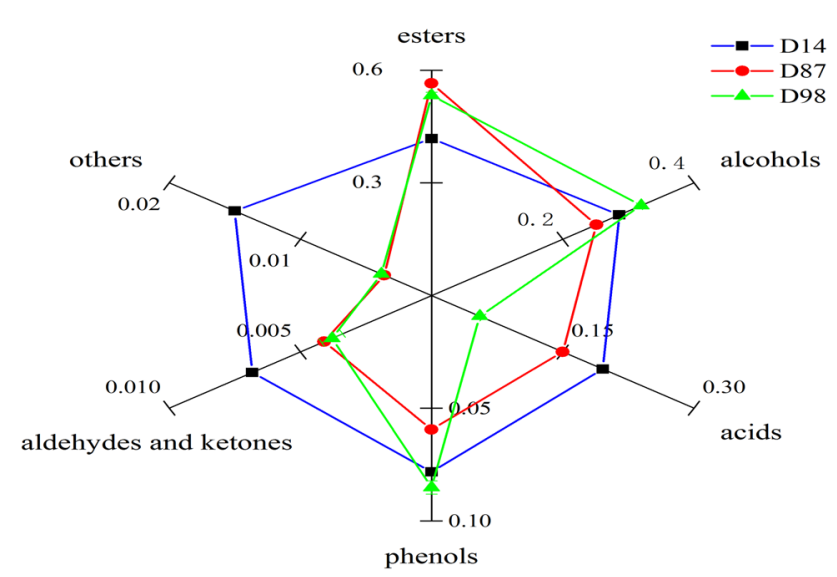

Fig. 4 Proportions of volatile components (esters, alcohols, acids, phenols, aldehydes, and ketones, and others) in the fermented grains of D14, D87, and D98 samples, respectively

$40-57 \%$ of all flavor components, which were dominant, followed by alcohols (25-32\%) and acids (5-20\%). The contents of esters and alcohols increased, while a noteworthy decrease of the acids, aldehydes and ketones, and others contents was observed along with the fermentation time.

Heatmap analysis of flavor components in fermented grains suggested that D98 sample fell into an alone cluster, while D14 and D87 samples were classified into another cluster (Fig. 5). Ethyl acetate (21.49\%), ethyl acetate (32.96\%), and diethyl succinate $(21.74 \%)$ were the most abundant ester in D14, D87, and D98 samples, respectively. The ethyl acetate content increased along with the extension of fermentation time from D14 to D87 samples, followed by a significant decline (32.96-9.36\%) from D87 to D98 samples. While the content of diethyl succinate had a marked increase (9.19-21.74\%) from D14 to D98 samples. Except ethanol, the most abundant alcohol was phenylethyl alcohol accounting for $19.98 \%, 10.47 \%$, and $16.60 \%$ in the fermented grains of D14, D87, and D98 samples, respectively, while isoamyl alcohol as the second most abundant alcohol, which accounted for $6.03 \%, 4.5 \%$, and $9.51 \%$ in these above-mentioned corresponding samples, respectively. The most abundant acid in fermented grains was acetic acid accounting for $14.34 \%, 13.50 \%$, and $2.54 \%$ in these aforementioned samples, respectively, which obviously decreased with the extended fermentation time. In addition, the hexadecanoic acid ethyl ester, linoleic acid ethyl ester, 2-butanol, and n-propanol contents increased with the extension of fermentation time.

\section{Correlation analysis between dominant microbes and volatile components}

The correlation network analysis indicated that 23 different volatile compounds were correlated with 14 dominant 
Fig. 5 Heatmap of flavor components in the fermented grains of three typical samples
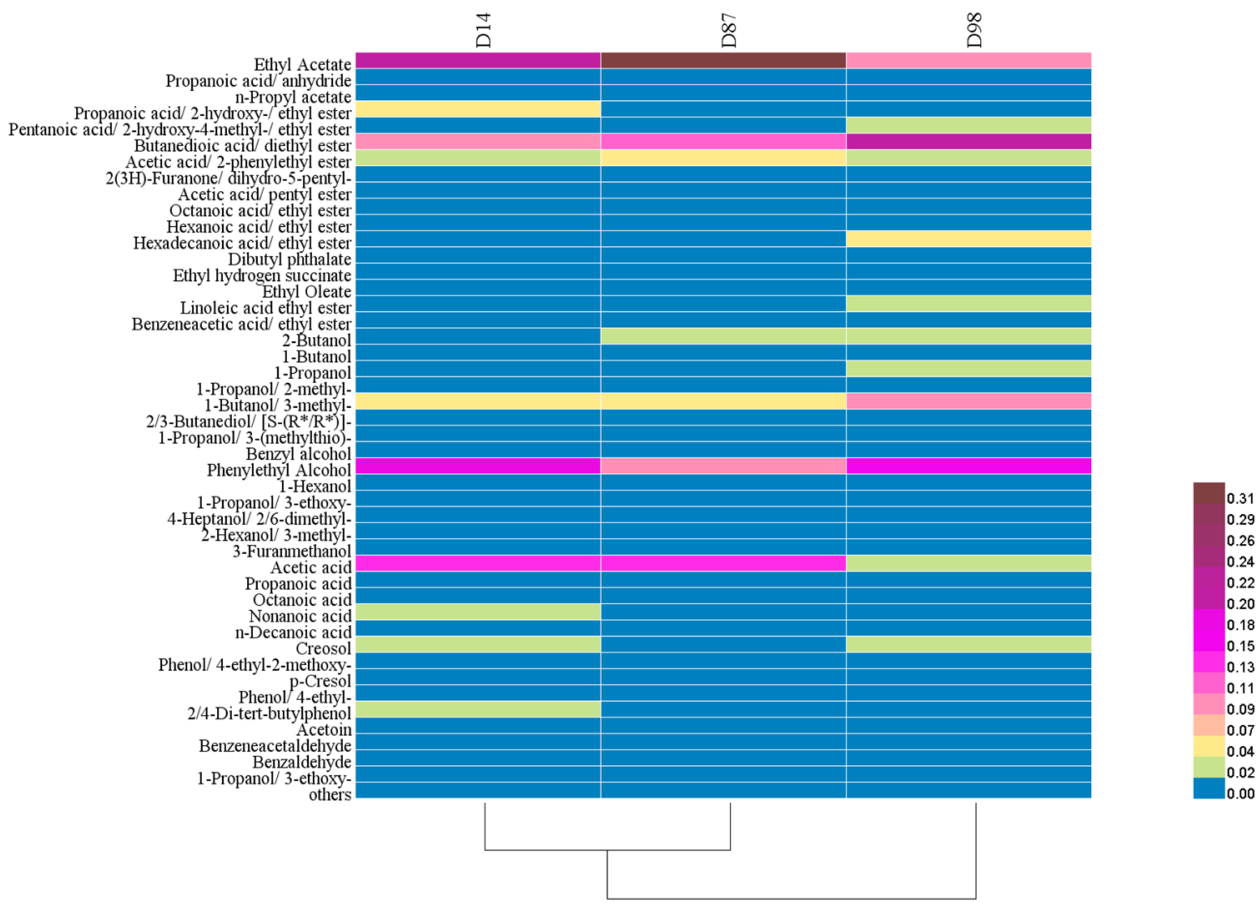

fungal genera (Fig. 6a) and 28 different volatile substances were correlated to 15 dominant bacterial species (Fig. 6b). Ethyl acetate, as the most abundant ester, displayed positive correlation with Cyberlindnera, Kazachstania, Candida, Lactobacillus sicerae, and Lactobacillus vini. The second most abundant diethyl succinate had positive correlation with Lactobacillus panis, Lactobacillus secaliphilus and negative correlation with Aspergillus. Phenylethyl alcohol showed negative correlation with L. vini and Lactobacillus hilgardii. Acetic acid with the most abundant in acids indicated positive correlation with Aspergillus and negative correlation with Trichoderma, Lactobacillus pontis, and $L$. helveticus.

Higher alcohols, mainly including n-propanol, isobutyl alcohol, and isoamyl alcohol, were one class of the main flavor components in Baijiu and the proper contents contributed to the formation of the rich flavor of Baijiu (Hu et al. 2021c). Isobutyl alcohol displayed positive correlation with Saccharomyces and Condenascus. Isoamyl alcohol, the second most abundant alcohols, had negative correlation with Kazachstania, Candida, Cyberlindnera, L. sicerae, and $L$. $v i n i$, and these microbes were positively correlated with ethyl acetate. The n-propanol, as the significantly increased substance, displayed negative correlation with Aspergillus and positive correlation with an uncultured Lactobacillus sp. As illustrated in Fig. 3, the decrease in the relative content of Aspergillus and the increase in the relative content of an uncultured Lactobacillus sp. maybe resulted in a sharp increase of n-propanol content in the fermented grains with the extended fermentation time.

\section{Relationships between physicochemical properties and microbial communities}

It is well known that physicochemical properties (moisture content, acidity, amino nitrogen, residual starch, and sugar contents) are responsible for the microbial community changes that occur in microbial ecosystems (Jung et al. 2014). Therefore, RDA was used to further analyze the relationship between the microbial communities and physicochemical properties mentioned above. An acute angle represents a positive correlation, while an obtuse angle indicates a negative correlation between the species and physicochemical properties. Overall, the two axes explained $93.62 \%$ and $83.98 \%$ of the total variance for the bacterial (Fig. 7a, DCA =1.93) and fungal (Fig. 7b, DCA =2.11) communities differentiation, respectively, and it suggested remarkable correlations between the microbial communities and physicochemical properties. RDA showed residual starch and reducing sugar content had positive correlations with A. pasteurianus, L. acetotolerans, Wickerhamomyces, Saccharomyces, and Condenascus, and negative correlations with L. helveticus, L. buchneri, Pseudeurotium, Oidiodendron, and Sistotrema. While moisture, acidity, and amino nitrogen had the opposite correlations with these microbes.

Monte Carlo permutation test showed residual starch, acidity, and amino nitrogen contents $(p<0.05)$ were important factors affecting the microbial community (Table 2). Specifically, residual starch had significant positive correlation with A. pasteurianus, L. acetotolerans, Wickerhamomyces, Saccharomyces, and Condenascus. 
Fig. 6 Correlation network diagram among dominant fungal genera (a), bacterial species (b), and volatiles (Note: Microbes and volatiles are represented by green circle and yellow square modules, respectively; Positive and negative correlations among dominant genera are represented by solid and dotted edges, respectively) (a)

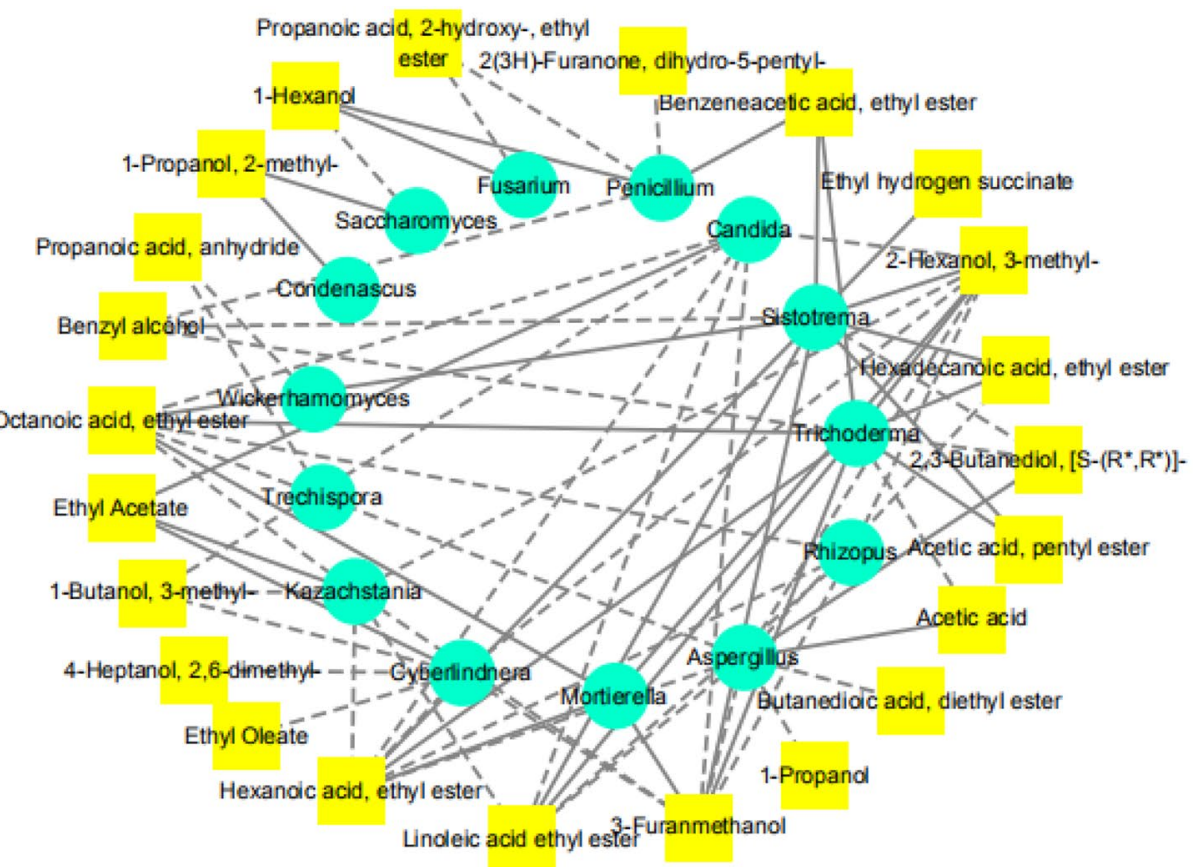

(b)

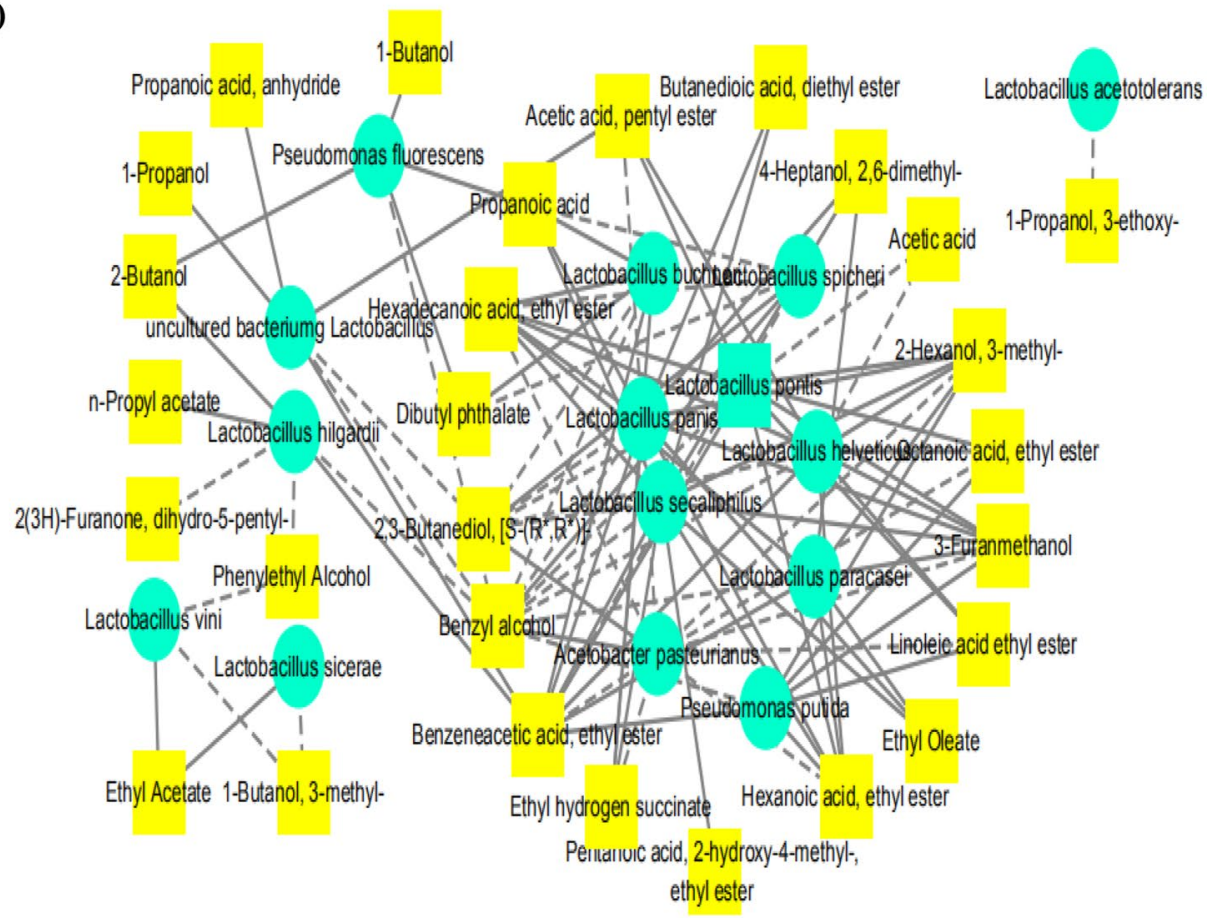

Acidity and amino nitrogen content showed significant positive correlations with $L$. helveticus, $L$. buchneri, uncultured Lactobacillus sp., Pseudeurotium, Oidiodendron, and Sistotrema. As illustrated in Fig. 2, the increase of acidity and amino nitrogen content and decrease of the residual starch content along with the extended fermentation time were related to the increase of $L$. helveticus, $L$. buchneri, uncultured Lactobacillus sp. and the reduction of A. pasteurianus, Wickerhamomyces, Saccharomyces, and Condenascus. 
Fig. 7 Redundancy analysis (RDA) of bacterial communities at species level (a) and fungal communities at genus level (b), and physicochemical properties (Note: blue arrows represent different physicochemical properties, red arrows represent different microbes; the symbols filled circle, filled square and filled diamond represent samples D14, D87, and D98, respectively) (a)

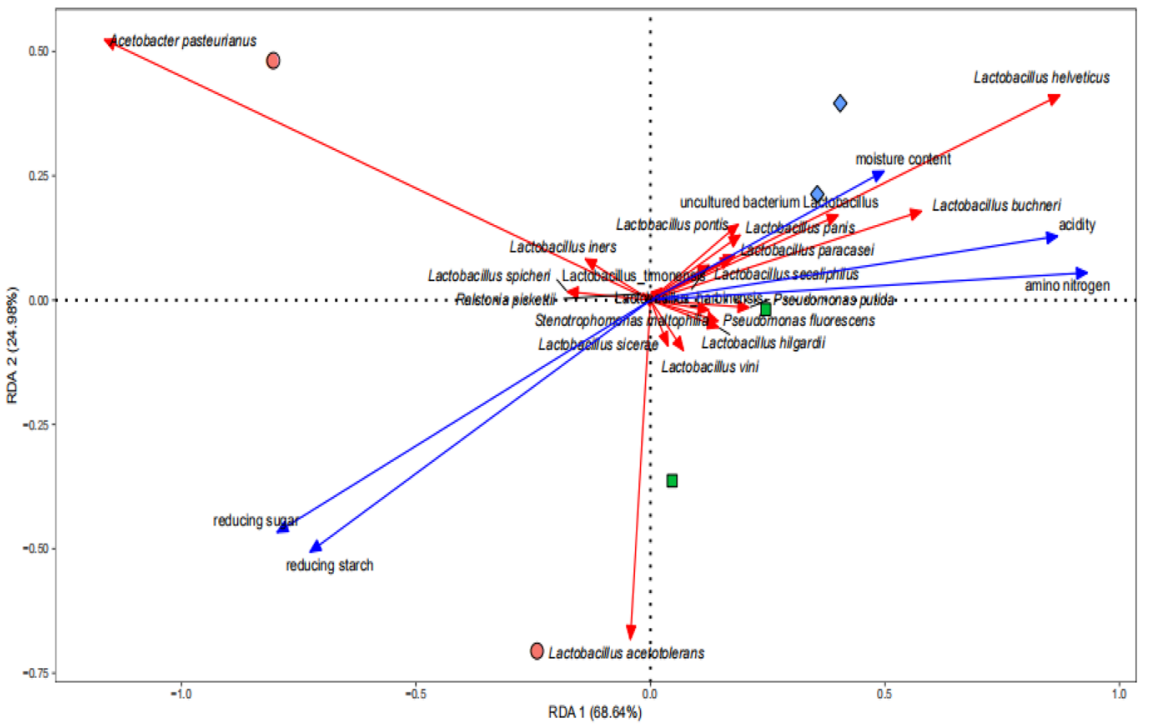

0.

(b)

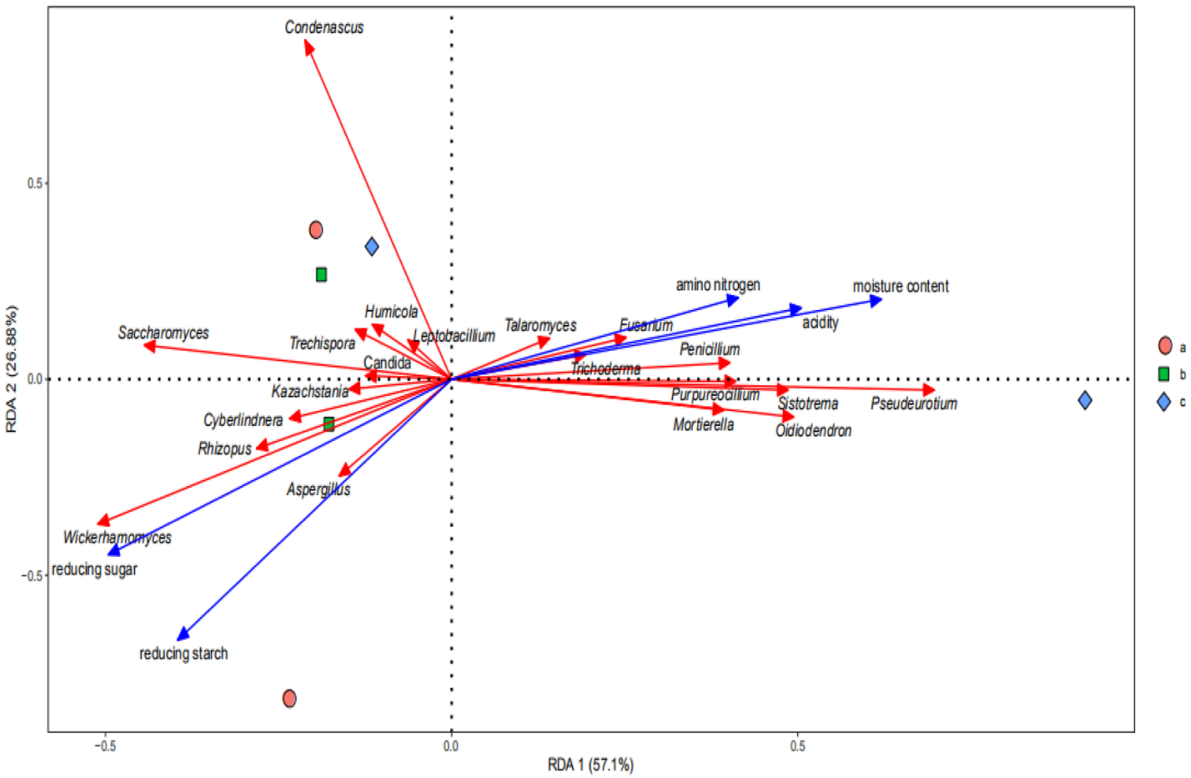

Table 2 Monte Carlo permutation test of the microbiota with physicochemical properties

\begin{tabular}{lll}
\hline Monte Carlo permutation test & $r^{2}$ & $p$ \\
\hline Moisture & 0.6948 & 0.15833 \\
Acidity & 0.8398 & $0.04167^{*}$ \\
Amino nitrogen & 0.8949 & $0.01528^{*}$ \\
Residual starch & 0.8974 & $0.02917^{*}$ \\
Reducing sugar & 0.6576 & 0.26667 \\
\hline
\end{tabular}

$* p<0.05$

\section{Discussion}

The brewing process of light-flavor Xiaoqu Baijiu is composed of some basic steps, including raw materials handling, grains steeping, grains steaming, starter preparation, cooling, grains blending, fermentation and distillation, etc. (Wang et al. 2020b). As one of the most important brewing processes, fermentation is performed by the synergistic effect of various microorganisms present in the fermented grains ecosystem, consisting of molds, yeasts, and bacteria (Jung et al. 2014). Up till now, the characterization of the microbial communities of fermented grains is being elucidated with the development of next-generation highthroughput sequencing technology (Huang et al. 2020; Guan et al. 2020; Liu and Miao 2020). But there are relatively few 
studies on the relationship between environmental factors and microbial community composition in fermented grains due to the limitation of sampling from the closed fermentation process, especially the effects of ultra-long fermentation time on the microbial community, physicochemical factors, and flavor components in fermented grains are rarely studied. To the best of our knowledge, this study, for the first time, analyzed the relationships between microbes and physicochemical properties or flavor components of fermented grains at the end of light-flavor Xiaoqu Baijiu fermentation from different fermentation periods.

In general, the coupling of HTS and plate counting technology provides a clear overview of microbial population (Huang et al. 2020). In our study, second-generation and third-generation amplicon technologies were used to describe the fungal and bacterial diversity of light-flavor Xiaoqu Baijiu, respectively. Figure 3 showed that dominant bacterial at the species level included L. acetotolerans, $A$. pasteurianus, L. helveticus, and L. buchneri, and dominant fungi at the genus level were Wickerhamomyces, Saccharomyces, Condenascus, Aspergillus, and Rhizopus in D14, D87, and D98 samples. Wang and Xu (2019), and Huang et al. (2020) revealed Bacillus and yeast were the most influential microorganisms in the early stage of fermentation, and lactic acid bacteria predominated in the late fermentation phase of light-flavor Baijiu. Hu et al. (2021b) used HTS to investigate the structure of the microbial community and indicated that the core microbes were Lactobacillus, A. pasteurianus, S. cerevisiae, W. anomalus, Aspergillus, and $R$. oryzae at the end of fermentation in the mechanized light-flavor Xiaoqu Baijiu production, which were basically in line with our results. But we newly found a fungus Condenascus that has not been reported in Baijiu, which formerly belonged to the classification of Thielavia (Wang et al. 2019a).

Compared to common fermentation time (day 14), the abundance of fungi (Wickerhamomyces, Saccharomyces, Condenascus, Aspergillus, and Rhizopus) decreased, and the abundance of lactic acid bacteria ( $L$. acetotolerans, $L$. helveticus, L. buchneri, and uncultured Lactobacillus sp.) increased to be dominant in the samples of ultra-long fermentation time (Fig. 3c, d). This was consistent with the results of culture-dependent analyses (Fig. 2). Previous research revealed that yeast population accounted for $45 \%$ of fungal abundance, followed by the LAB in the sample of normal fermentation time (day 14) (Hu et al. 2021c). But ultra-long fermentation time resulted in the increase of $\mathrm{LAB}$ and the decrease of yeasts, which were due to the changes of environmental factors, including nutrient limitation, high ethanol concentration, and low $\mathrm{pH}$ value (As showed in Fig. 1 and Fig. 2). For instance, A. pasteurianus was subdominant in D14 sample, but its relative abundance was less than 1\% in D87 sample. And Lactobacillus dominated in
D98 (96.28\%) sample. The proliferation of LAB and production of lactic acid may inhibit the growth of A. pasteurianus.

In this study, RDA was used to analyze the correlation between physicochemical properties and microbial community of light-flavor Xiaoqu Baijiu. The results revealed that residual starch and reducing sugar content had positive correlations with A. pasteurianus, L. acetotolerans, Wickerhamomyces, Saccharomyces, and Condenascus, and negative correlations with L. helveticus, L. buchneri, Pseudeurotium, Oidiodendron, and Sistotrema. While moisture, acidity, and amino nitrogen had the opposite correlations with these microbes. Furthermore, mantel tests showed that changes in residual starch, acidity, amino nitrogen had significant effects on microbial composition. Previous studies demonstrated high concentrations of water, ethanol as well as high acidity could facilitate the growth of Lactobacillus in the brewing process (Liu and Miao 2020). Therefore, the high acid environment in the ultra-long fermentation sample (D98) formed a micro-environment that was conducive to the growth of Lactobacillus.

Flavor components are very important indicators for Baijiu quality, which are largely affected by the microbes in fermentation micro-ecosystem (Wang et al. 2019b). The content of esters (such as ethyl acetate, diethyl succinate, phenylethyl acetate, ethyl palmitate, and ethyl linoleate) and alcohols (isoamyl alcohol and n-propanol) increased and the acids content (acetic acid) reduced significantly along with the fermentation progressed (Fig. 4). The later stage of fermentation process in fermented grains (25-60 days) could be described as the period of esterification and acetic acid is a precursor substance of synthetic esters, which led to a rise in the content of esters and a reduction in acids $(\mathrm{Hu}$ et al. 2021a). Ethyl acetate as main ester, is recognized as a key compound in light-flavor Baijiu. Although prolonging the fermentation time was beneficial to the accumulation of ethyl acetate, ultra-long fermentation time will cause its content to decrease, and other esters, such as diethyl succinate, accounted for the most part of esters in D98 sample (Fig. 5). Previous studies indicated that yeasts, bacteria, and molds took part in the synthesis of ethyl acetate (Guo and Jia 2014). In this study, ethyl acetate displayed positive correlation with Cyberlindnera, Kazachstania, Candida, L. sicerae, and $L$. vini, and diethyl succinate showed negative correlation with Aspergillus and positive correlation with L. panis, L. secaliphilus (Fig. 6). It indicated Lactobacillus contributed much in esters metabolism in light-flavor Baijiu fermentation, and Pang et al. (2021) revealed that inoculation with indigenous LAB changed the flavor profile of Baijiu significantly, especially promoting the formation of ethyl acetate. Besides, acetic acid and phenylethyl alcohol were the main aromatic compounds of Baijiu (Wang and Xu 2019). Acetic acid reduced significantly, and phenylethyl alcohol kept a relatively stable content along with the extension of 
fermentation time from 14 to 98 days. Correlation analysis showed that acetic acid positively correlated with Aspergillus and negatively correlated with Trichoderma, L. pontis, and L. helveticus (Fig. 6). In the late phrase of fermentation, LAB became the dominant species under anaerobic condition, and restrained the reproduction of Acetobacter by niche occupation. Thus, acetic acid was observed to decrease significantly in the long-time fermentation progress.

Higher alcohols, mainly including n-propanol, isobutyl alcohol, and isoamyl alcohol, are produced as the secondary metabolites of yeast during alcoholic fermentation (Wang et al. 2020c). At moderate level, these flavor components contribute to the flavor of many alcoholic beverages, whereas the excess level may be a health hazard ( $\mathrm{Hu}$ et al. 2021c). Therefore, researchers have studied how to control the production of higher alcohols by process control and microbial regulation (Jiang et al. 2019; Pang et al. 2021; Zhong et al. 2019). In this study, the content of higher alcohols increased rapidly along with the extension of fermentation time, especially the n-propanol content increased by 6 times in fermented grains of D98 sample, compared to that in the D14 sample. Correlation network showed that $\mathrm{n}$-propanol was positive correlation with an uncultured $\mathrm{Lac}$ tobacillus $\mathrm{sp}$., which was regard as one of major microbes for n-propanol production in Baijiu fermentation (Lu et al. 2019; Tian et al. 2020). Overall, microbial community had a significant variation induced by the ultra-long time fermentation, especially bacterial community. Lactobacillus accounted for the most part of microbes in fermented grains at the end of fermentation, which played a crucial role in the synthesis of key aroma compounds in fermented grains, such as ester, acid, alcohols, and so on.

Prolonging the fermentation time can improve the diverstiy of flavor components in Baijiu. For example, the fermentation time of strong-flavor Вaijiu and sauce-flavor Вaijiu is as long as 30 to 60 days, which is more likely to produce flavor components with diverse structural features and interesting biological activities, such as esters, acids, phenols, and pyrazines, which make Baijiu possesses the features of elegant and soft and endless aftertaste. However, ultra-long fermentation time also results in several potential side effects. First, it will lead to low liquor yield, this was encountered in strong-flavor Baijiu and sauce-flavor Baijiu. Another potential risk of ultra-long fermentation time is yielding more undesired components. In our case, ultra-long fermentation time is often accompanied by an increase in higher alcohols, especially n-propanol. Since the increase of LAB and ultra-long fermentation provide the possibility for the synthesis of n-propanol, which contributes negatively to the flavor of Baijiu when it is present in high concentrations. It is important to avoid the increase of the content of some components that cause human discomfort. In our study, the overall quality of Baijiu produced by 87 and 98 days fermentation was not as good as that of the normal fermentation (D14). For this reason, they were used as flavoring liquor to produce different styles of Baijiu. Therefore, in the future, designing the appropriate fermentation time is not only conducive to brewing the original Baijiu containing different flavor components, but also balances the flavor components and yield of liquor.

\section{Conclusion}

Our results provide an overview of effect of ultra-long fermentation time on microbial community, physicochemical properties, and flavor components of light-flavor Xiaoqu Baijiu based on the fermentation tanks. Long-time fermentation significantly changes microbial community and improves the diversity of volatile components via adjusting physicochemical parameters, compared to the normal fermentation. L. acetotolerans, L. helveticus, L. buchneri, Wickerhamomyces, Saccharomyces, and Condenascus were identified as dominant microbes, and Lactobacillus absolutely dominated at the end of ultra-long fermented grains, which accounted for $96.28 \%$ of the total bacteria in fermented grains of D98 sample. Volatile components were mainly influenced by the microbial succession during ultra-long fermentation process, such as the massive production of $\mathrm{n}$-propanol may be related to the proliferation of an uncultured Lactobacillus sp.. Residual starch, acidity, and amino nitrogen played important roles in promoting the formation of functional microbes. In a word, ultra-long fermentation time changes the overall flavor balance of Baijiu and improve the yielding of esters and alcohols of light-flavor Xiaoqu Baijiu, which are conducive to the production of special flavoring liquor. These results not only expanded our understanding on the factors that influenced the Baijiu quality, they may also provide a new strategy to breed new superior strains. We believe the quality of Baijiu can be stabilized by controlling the physicochemical changes influencing the succession of microbial communities and related microorganisms producing key flavor components well.

Acknowledgements We gratefully acknowledge financial support from the Hubei Provincial Key Research and Development Program (2020BBA050).

\section{Declarations}

Conflict of interest The authors have no conflicts of interest to report.

\section{References}

Amend A, Samson R, Seifert K, Bruns T (2020) Deep sequencing reveals diverse and geographically structured assemblages of fungi in indoor dust. Proc Natl Acad Sci USA 107:13748-13753

Bokulich NA, Subramanian S, Faith JJ, Gevers D, Gordon JI, Knight R, Caporaso JG (2013) Quality-filtering vastly improves diversity 
estimates from Illumina amplicon sequencing. Nat Methods 10:57-59

Cai HY, Zhang T, Zhang Q, Luo J, Cai CG, Mao JW (2018) Microbial diversity and chemical analysis of the starters used in traditional Chinese sweet rice wine. Food Microbiol 73:319-326

Dong WW, Yang Q, Liao YX, Liu YC, Hu YL, Peng N, Liang YX, Zhao SM (2020) Characterisation and comparison of the microflora of traditional and pure culture Xiaoqu during the Baijiu liquor brewing process. J Inst Brew 126:213-220

Fan GS, Fu ZL, Teng C, Liu PX, Wu QH, Rahman MKR, Li XT (2020) Effects of aging on the quality of roasted sesame-like flavor Daqu. BMC Microbiol 20:67

Guan TW, Lin YJ, Chen KB, Ou MY, Zhang JX (2020) Physicochemical factors affecting microbiota dynamics during traditional solidstate fermentation of Chinese strong-flavor Baijiu. Front Microbiol 11:2090

Guo JH, Jia SR (2014) Effects of enzymes on ester production during the course of a Chinese liquor fermentation as discussed by correlation analysis and path analysis. J Inst Brew 120:565-570

Hao F, Tan Y, Lv X, Chen LQ, Yang F, Wang H, Du H, Wang L, Xu Y (2021) Microbial community succession and its environment driving factors during initial fermentation of Maotai-Flavor Baijiu. Front Microbiol 12:669201

Hu XL, Tian RJ, Wang KL, Cao ZH, Yan PX, Li FQ, Li XS, Li SL, He PX (2021a) The prokaryotic community, physicochemical properties and flavors dynamics and their correlations in fermented grains for Chinese strong-flavor Baijiu production. Food Res Int 148:110626

Hu YL, Wang LY, Zhang ZJ, Yang Q, Chen SX, Zhang L, Xia X, Tu JM, Liang YX, Zhao SM (2021b) Microbial community changes during the mechanized production of light aroma Xiaoqu baijiu. Biotechnol Biotechnol Equip 35:487-495

Hu YL, Yang Q, Chen D, Fu B, Zhang Y, Zhang Y, Xia X, Peng N, Liang YX, Zhao SM (2021c) Study on microbial communities and higher alcohol formations in the fermentation of Chinese Xiaoqu Baijiu produced by traditional and new mechanical technologies. Food Res Int 140:109876

Huang XN, Fan Y, Lu T, Kang JM, Pang XN, Han BZ, Chen JY (2020) Composition and metabolic functions of the microbiome in fermented grain during light-flavor Baijiu fermentation. Microorganisms 8:1281

Huson DH, Richter DC, Rausch C, Dezulian T, Franz M, Rupp R (2007) Dendroscope: an interactive viewer for large phylogenetic trees. BMC Bioinform 8(1):1-6

Jiang J, Liu YC, Li HH, Yang Q, Wu Q, Chen SX, Tang J, Xu Y (2019) Modeling and regulation of higher alcohol production through the combined effects of the $\mathrm{C} / \mathrm{N}$ ratio and microbial interaction. $\mathrm{J}$ Agric Food Chem 67:10694-10701

Jin G, Zhu Y, Xu Y (2017) Mystery behind Chinese liquor fermentation. Trends Food Sci Technol 63:18-28

Jung JY, Lee SH, Jeon CO (2014) Microbial community dynamics during fermentation of doenjang-meju, traditional Korean fermented soybean. Int J Food Microbiol 185:112-120

Lee SM, Kim SB, Kim YS (2019) Determination of key volatile compounds related to long-term fermentation of soy sauce. J Food Sci 84(10):2578-2776

Li XR, Ma EB, Yan LZ, Meng H, Du XW, Zhang SW, Quan ZX (2011) Bacterial and fungal diversity in the traditional Chinese liquor fermentation process. Int J Food Microbiol 146:31-37

Liu PL, Miao LH (2020) Multiple batches of fermentation promote the formation of functional microbiota in Chinese MiscellaneousFlavor Baijiu fermentation. Front Microbiol 11:75

Liu PL, Xiong XM, Wang S, Miao LH (2017) Population dynamics and metabolite analysis of yeasts involved in a Chinese miscellaneous-flavor liquor fermentation. Ann Microbiol 67:553-565
Liu CC, Feng SB, Wu Q, Huang HQ, Chen ZX, Li SW, Xu Y (2019) Raw material regulates flavor formation via driving microbiota in Chinese liquor fermentation. Front Microbiol 10:15-20

Lu JJ, Yang F, Yang J, Chen LQ, Liu YF, Wang HY, Wang L (2019) Source tracing of 1-propanol-producing microorganisms during Baijiu fermentation. China Brewing 38(7):151-155 ((in Chinese))

Pang XN, Chen C, Huang XN, Yan YZ, Chen JY, Han BZ (2021) Influence of indigenous lactic acid bacteria on the volatile flavor profile of light-flavor Baijiu. LWT Food Sci Technol 147:111540

Quast C, Pruesse E, Yilmaz P, Gerken J, Schweer T, Yarza P, Glöckner FO (2012) The SILVA ribosomal RNA gene database project: Improved data processing and web-based tools. Nucleic Acids Res 1:590-596

Quijada NM, Filippis DF, Sanz JJ, García-Fernández MC, Rodríguez LD, Ercolini D, Hernández M (2017) High-throughput sequencing analysis reveals different Lactobacillus populations that dominate during "Chorizo de León" cured meat making. Food Microbiol 70:94-102

Ramette A (2007) Multivariate analyses in microbial ecology. FEMS Microbiol Ecol 62:142-160

Shannon P, Markiel A, Ozier O, Baliga NS, Wang JT, Ramage D, Amin N, Schwikowski B, Ideker T (2003) Cytoscape: a software environment for integrated models of biomolecular interaction networks. Genome Res 13:2498-2504

Sudhanshu SB, Ramesh CR, Nevijo Z (2018) Lactobacillus plantarum with functional properties: an approach to increase safety and shelf-life of fermented foods. Biomed Res Int. https://doi.org/10. 1155/2018/9361614

Tan YW, Zhong HP, Zhao D, Du H, Xu Y (2019) Succession rate of microbial community causes flavor difference in strong-aroma Baijiu making process. Int J Food Microbiol 311:108350

Tian Y, Kong XY, Fang F (2020) Microbial n-propanol synthesis during Luzhou-flavor liquor fermentation. Acta Microbiol Sin 60:1421-1432 ((in Chinese))

Wang HY, Xu Y (2019) Microbial succession and metabolite changes during the fermentation of Chinese light aroma-style liquor. J Inst Brew 125:162-170

Wang XW, Bai FY, Bensch K, Meijer M, Sun BD, Han YF, Crous PW, Samson RA, Yang FY, Houbraken J (2019a) Phylogenetic re-evaluation of Thielavia with the introduction of a new family Podosporaceae. Stud Mycol 93:155-252

Wang S, Wu Q, Nie Y, Wu J, Xu Y (2019b) Construction of synthetic microbiota for reproducible flavor compound metabolism in Chinese light-aroma-type liquor produced by solid-state fermentation. Appl Environ Microbiol 85:e03090-e3118

Wang W, Fan G, Li X, Fu Z, Liang X, Sun B (2020a) Application of Wickerhamomyces anomalus in simulated solid-state fermentation for Baijiu production: changes of microbial community structure and flavor metabolism. Front Microbiol 11:598-758

Wang Z, Su ZX, Yang Q, Liu YC, Lin B, Chen SX, Yang H (2020b) Characterizing relationship of microbial community in Xiaoqu and volatiles of light-aroma-type Xiaoqu Baijiu. Food Sci Technol Res 26:749-758

Wang CX, Tang JD, Qiu SY (2020c) Profiling of fungal diversity and fermentative yeasts in traditional Chinese Xiaoqu. Front Microbiol 11:2103

Ye H, Wang J, Shi J, Du J, Zhou Y, Huang M, Sun B (2021) Automatic and intelligent technologies of solid-state fermentation process of Baijiu production: applications, challenges, and prospects. Foods 10:680

Zang JH, Xu YS, Xia WS, Yu DW, Gao P, Jiang QX, Yang F (2018) Dynamics and diversity of microbial community succession during fermentation of Suan yu, a Chinese traditional fermented fish, determined by high throughput sequencing. Food Res Int 111:565-573 
Zhang HX, Wang L, Wang HY, Yang F, Chen LQ, Hao F, Lv XB, Du $\mathrm{H}, \mathrm{Xu}$ Y (2021) Effects of initial temperature on microbial community succession rate and volatile flavors during Baijiu fermentation process. Food Res Int 141:109887

Zhao M, Su XQ, Nian B, Chen LJ, Zhang DL, Duan SM, Wang LY, Shi XY, Jiang B, Jiang WW (2019) Integrated meta-omics approaches to understand the microbiome of spontaneous fermentation of traditional Chinese Pu-erh tea. mSystems 4:e00680-19

Zheng XW, Han BZ (2016) Baijiu (白酒), Chinese liquor: History, classification and manufacture. J Ethn Foods 3:19-25
Zhong XF, Wang AL, Zhang YB, Wu ZY, Li B, Lou H, Huang GD, Wen HX (2019) Reducing higher alcohols by nitrogen compensation during fermentation of Chinese rice wine. Food Sci Biotechnol 29:805-816

Publisher's Note Springer Nature remains neutral with regard to jurisdictional claims in published maps and institutional affiliations. 